\title{
QUAIS FATORES ESTÃO MAIS RELACIONADOS COM A SATISFAÇÃO COM O TRABALHO E O COMPROMETIMENTO ORGANIZACIONAL NO IFPA - CAMPUS SANTARÉM?
}

\author{
WHAT FACTORS ARE MOST RELATED WITH SATISFACTION \\ WORK AND ORGANIZATIONAL COMMITMENT AT FEDERAL \\ INSTITUTE IN PARÁ STATE IN BRAZIL - CAMPUS SANTARÉM? \\ ¿CUÁLES FACTORES ESTÁN MÁS RELACIONADOS CON \\ LA SATISFACCIÓN CON EL TRABAJO Y EL COMPROMISO \\ ORGANIZACIONAL EN EL IFPA - CAMPUS SANTARÉM?
}

Luiz Honorato da Silva Júnior

Fernanda Regina Nascimento

Maria José Buchalle Silva

Lucijane Monteiro de Abreu

RESUMO: Este trabalho busca evidências a respeito dos fatores que influenciam o comprometimento organizacional e a satisfação no trabalho de servidores do Instituto Federal do Pará, Campus Santarém. Fizeram parte da pesquisa a totalidade dos servidores docentes e técnicos administrativos do referido Campus, totalizando 68 servidores que responderam a aplicação de um questionário. Foram propostos indicadores para mensurar o nível de Comprometimento e Satisfação dos servidores e, em seguida, regressões em Mínimos Quadrados Ordinários com o objetivo de se compreender quais fatores estariam mais relacionados com os coeficientes. Os resultados trouxeram evidências de que os servidores mais satisfeitos são àqueles mais comprometidos com o trabalho e a recíproca também verdadeira. Servidores mais jovens e de menor escolaridade eram aqueles que revelavam menor comprometimento, enquanto que os servidores com maior escolaridade associado a um maior tempo de serviço revelam maior nível de comprometimento. Da mesma maneira, servidores mais velhos revelaram maior satisfação, assim como os técnicos administrativos revelaram maior satisfação que os docentes. Esses resultados podem subsidiar políticas que promovam ações que aperfeiçoem a eficiência da administração pública.

PALAVRAS-CHAVE: Comprometimento organizacional. Satisfação no Trabalho. IFPA.

ABSTRACT: This paper seeks evidence about the factors that influence the organizational commitment and Labor satisfaction of the servers at Federal Institute of Pará, Santarém Campus. The participants were all the teachers and administrative staff of servers that Campus, totaling 68 servers that responded to the application of a questionnaire. Indicators were proposed that were intended to measure the level of commitment and satisfaction of the servers and then made regressions OLS in order to understand which factors are more related to the coefficients. The results brought evidence that the most satisfied servers are those most committed to the work and the reciprocal was also true. Younger servers and less education were those who showed less commitment, while the servers with higher education associated with greater service time show higher level of commitment. Similarly, older servers 
showed higher satisfaction, as well as administrative staff showed greater satisfaction than teachers. These results may support policies that promote actions that improve the efficiency of public administration.

KEYWORDs: Organizational commitment. Labor Satisfaction. Federal Institute of Pará.

RESUMEN: Este trabajo busca evidencias acerca de los factores que influencian el compromiso organizacional y la satisfacción en el trabajo de servidores del Instituto Federal de Pará, Campus Santarém. Se realizaron parte de la investigación la totalidad de los servidores docentes y técnicos administrativos del referido Campus, totalizando 68 servidores que respondieron a la aplicación de un cuestionario. Se propusieron indicadores para medir el nivel de Compromiso y Satisfacción de los servidores y luego regresiones en Mínimos Cuadrados Ordinarios con el objetivo de comprender qué factores estarían más relacionados con los coeficientes. Los resultados arrojaron evidencias de que los servidores más satisfechos son los más comprometidos con el trabajo y la recíproca también verdadera. Los servidores más jóvenes y de menor escolaridad eran aquellos que revelaban un menor compromiso, mientras que los servidores con mayor escolaridad asociados a un mayor tiempo de servicio revelan un mayor nivel de compromiso. De la misma manera, servidores más viejos revelaron mayor satisfacción, así como los técnicos administrativos revelaron mayor satisfacción que los docentes. Estos resultados pueden subsidiar políticas que promuevan acciones que mejoren la eficiencia de la administración pública.

PAlAbras Clave: Compromiso organizacional. Satisfacción en el trabajo. IFPA. 


\section{INTRODUÇÃO}

Muitos são os questionamentos que permeiam a discussão a respeito da satisfação e do comprometimento no trabalho de servidores públicos no Brasil. Muito se discute sobre a relação existente entre ambas, ou seja, se servidores mais satisfeitos seriam também os mais comprometidos com o trabalho e vice-versa. Além disso, pergunta-se: quais características associadas aos servidores são mais relacionadas com essas percepções acerca do trabalho? Características como sexo, idade, cargo, tempo de serviço, entre outras características, influenciariam nessa percepção de satisfação e de comprometimento?

A impressão que se tem no dia-a-dia, sobretudo quem frequenta as chamadas "repartições públicas brasileiras", é a de que se tem um enorme número de servidores insatisfeitos com a atividade e um diminuto comprometimento no trabalho, sobretudo, quando a medida de comparação é o setor privado da economia. Entretanto, é importante ressaltar, que se trata de percepções à priori, e é difícil afirmar qualquer coisa a respeito, porque não existem medidores objetivos dessas percepções.

Tais constatações reforçam a necessidade da realização de estudos sobre o comportamento dos servidores públicos, tendo em vista que o nível de satisfação e comprometimento define a qualidade do trabalho desempenhado.

Portanto, este trabalho tem como principal objetivo buscar evidências a respeito do nível de satisfação e comprometimento no trabalho revelados pelos servidores do Instituto Federal do Pará - Campus Santarém. Pretende-se, entender quais fatores relacionados a esses servidores estariam mais relacionados com a satisfação e o comprometimento no trabalho. Buscam-se evidências sobre quais características diretamente observáveis dos servidores estariam mais relacionadas com servidores mais satisfeitos e comprometidos com o trabalho.

O atendimento dos objetivos desta pesquisa pode contribuir diretamente com o aperfeiçoamento da gestão no IFPA Campus Santarém e ainda, contribuir para a rica discussão acadêmica sobre o tema e trazer resultados de ganho de produtividade, além de intervenções na política de gestão de pessoas do IFPA.

\section{COMPROMETIMENTO ORGANIZACIONAL E SATISFAÇÃO NO TRABALHO}

O advento da globalização a partir da década de noventa provocou alterações na gestão das empresas, de forma que estas tiveram que se adequar às novas tendências para continuarem competitivas no mercado globalizado.

Tais tendências trouxeram, entre outras prioridades, um novo olhar para os recursos humanos, que começaram a ser vistos como importante capital para as organizações e que precisavam compor o Capital Intelectual das empresas. 
Segundo Dessler (1996) é necessário mais do que funcionários comprometidos - que se identificam com a empresa e adotam suas metas como se fossem seus proprietários. Para o autor cada vez mais, funcionários comprometidos é a chave para o diferencial competitivo da empresa.

Leite (2004) ressalta que as instituições públicas não fogem a esse contexto, pois essas tendências fizeram também, com que a sociedade passasse a exigir mais das instituições. Ainda para esse autor, o comprometimento tem sido entendido por vários pesquisadores como uma espécie de laço psicológico que caracteriza o relacionamento entre o indivíduo e a organização.

Os estudos sobre o elo que se estabelece entre indivíduo e organização tem demonstrado crescente interesse de pesquisadores ligados a diferentes áreas, como a psicologia, sociologia e teoria das organizações.

Gomes e Quelhas (2003 afirmam que o governo Federal, desde 1990, tem se esforçado para profissionalizar a gestão pública. Apesar disso, continua focada na impessoalidade e tecnicismo, desconsiderando o fator emocional de cada um, subestimando sua potencialidade mental e sua percepção do ambiente a sua volta, visto que isso refletirá e muito na sua motivação e satisfação no trabalho.

Vale lembrar ainda que, outras questões como motivação, identidade pessoal e organizacional, liderança, entre outros; são fatores presentes na organização e influenciam diretamente o desenvolvimento pessoal e a produtividade da organização e, além disso, estão diretamente ligados ao comprometimento e satisfação de cada um.

No trabalho de Etizione (1961), o comprometimento é abordado como um conjunto de pressões internalizadas pelo indivíduo, de tal forma que este se comporte convenientemente no cumprimento dos objetivos e interesses organizacionais.

Em sua meta-análise, Mathieu \& Zajac (1990), citado por Moraes \& Dias (1993), Bastos (1993), Brandão (1991) apontam um conjunto de dimensões, indicadoras dos antecedentes do comprometimento organizacional. Este conjunto de elementos é categorizado nas seguintes variáveis: características pessoais, do trabalho, relações líder-grupo, características organizacionais e do papel. Quanto às características pessoais, as investigações desses autores evidenciaram correlações entre estas e o comprometimento.

Maior nível de comprometimento é estabelecido por indivíduos como maior tempo na organização e de maior idade, segundo os trabalhos de Angle \& Perly (1981), Krebiniak (1974), Morris \& Cherman (1981), citados por Moraes \& Dias (1993). Além disso, outros aspectos como remuneração mais elevada, trabalhadores do sexo masculino e maior nível educacional parecem determinar maiores níveis de comprometimento segundo esses autores.

Por sua vez, Mowday (1982) aponta que existe uma correlação negativa entre comprometimento e o nível educacional. Tal fato se 
justificaria uma vez que, os indivíduos de escolaridade mais elevada trazem maior expectativa e nem sempre as organizações estão aptas a supri-las.

Baixas correlações entre as características pessoais (idade, numero de filhos, estado civil, tempo de serviço), nível de habilidade, percepção de alternativas de trabalho e sistema de pagamentos foram encontrados por Cohen \& Lowberg, citados por Bastos (1993).

Indicadores como a variabilidade, identidade e o significado das tarefas, inter-relacionamento, feedback do trabalho e dos colegas e a autonomia tem sido aplicado com o objetivo de verificar tais correlações e apresentam-se como forte preditores do comprometimento no trabalho de Monday et al (1982) e Motaz (1988), citados por Brito (2000).

Borges-Andrade (1989) e Brandão (1991) chamam a atenção para tendência explicativa das variáveis organizacionais, especialmente no que se refere a oportunidade de crescimento e realização profissional oferecida ao servidor.

Ao aplicar o modelo da técnica comprobatória Willian e Hazer citados por Moraes \& Dias (1993), defendem a existência de evidências empíricas que mostram que a satisfação no trabalho é uma causa do comprometimento organizacional. Tal afirmativa vai ao encontro com as premissas de Bluerdon (1982), Koch e Steers (1977).

Para Amâncio et al. (2014) relata que, apesar da subjetividade do tema, a satisfação no trabalho é o conjunto de sentimentos favoráveis que o indivíduo demonstra em relação ao trabalho e isso pode influenciar de forma positiva o seu empenho. Vale ressaltar que "a intensidade da satisfação e a importância de valores não podem ser medidos em termos de quaisquer unidades físicas ou psicológicas conhecidas" (Locke, 1969, p.331). No entanto, não considerar que esses conceitos não são significativos seria um erro.

A relação entre comprometimento organizacional e a satisfação do indivíduo, enquanto força de trabalho, é também evidenciada por Moraes e Dias (1993), citado por Brito (2000). Ao executarem um diagnostico do comprometimento dos servidores de uma organização publica, ligada ao serviço de extensão, encontram relação direta entre as variáveis do contexto de trabalho (níveis de satisfação com segurança, compensação, possibilidade de crescimento, satisfação salarial com referência a equidade interna e externa), que explicam $40 \%$ do grau de comprometimento.

Os estudos iniciais sobre satisfação no trabalho remontam ao período dos anos vinte e tinham por objetivo estudar situações de fadiga e monotonia dos trabalhadores. Nesse período surgem as primeiras preocupações científicas sobre a influência das condições físicas do local de trabalho na produção industrial.

Segundo Coda (1997), citado no artigo de Pinto (2015) a ideia de que a satisfação no trabalho influencia as decisões das pessoas sobre trabalhar e de permanecerem no atual emprego ainda parece ser válida. Cada vez mais as organizações conduzem levantamento de atitudes 
no trabalho para identificar os sentimentos de satisfação de seus colaboradores, para entender como a organização opera e para avaliar os efeitos de práticas gerenciais específicas e de arranjos organizacionais.

Autores como Maslow (1970) e Henzberg (1973) contribuíram significativamente para estudos nessa área. Maslow baseia seus estudos na teoria da hierarquia das necessidades humanas. Henzberg estuda sua teoria baseada nos fatores higiênicos e de motivação.

A partir das considerações acima, sobre comprometimento organizacional, verifica-se que o tema é amplo, complexo, multidisciplinar, investigativo e requer aprofundamentos em novos trabalhos para o avanço de outras pesquisas na área.

\section{METODOLOGIA DA PESQUISA}

Os dados necessários à pesquisa foram produzidos a partir da elaboração e aplicação de um questionário junto a servidores técnicos administrativos e docentes lotados no IFPA - Campus Santarém. No total foram aplicados 68 questionários alcançando assim, a totalidade de servidores lotados naquele Campus.

O referido questionário foi aplicado no período entre dezembro de 2015 a janeiro de 2016 e o objetivo desse instrumento foi captar a percepção dos servidores do Campus Santarém com relação ao comprometimento organizacional e a satisfação no trabalho.

No referido questionário continha perguntas com a finalidade de se identificar algumas das principais características sociais do servidor, como sexo, idade, escolaridade, etc, e que na sequência seriam correlacionadas com seus respectivos coeficientes de Comprometimento e de Satisfação, conforme detalhado em seguida.

Em seguida, o questionário arguia o respondente sobre aspectos que permitiram construir o coeficiente de comprometimento e de satisfação no trabalho. Esta parte foi composta por perguntas fechadas com duas ou três opções de respostas.

Uma vez confeccionado o questionário foram realizados pré-testes com a finalidade de adequar melhor as perguntas, com o intuito de dirimir as dúvidas em relação à clareza e entendimento das perguntas para somente depois os dados serem coletados.

\subsection{FORMULAÇÃO DOS COEFICIENTES}

Com o objetivo de se tentar mensurar o comprometimento e a satisfação dos servidores, propuseram-se coeficientes que dessem uma medida dessas duas características. Assim, foi proposto um indicador que garanta a agregação de valores a partir de respostas que estejam diretamente ligadas com o crescimento daquela variável. 
O primeiro coeficiente propõe capitar o efeito do comprometimento dos respondentes, da seguinte maneira:

$$
\mathbf{C C}=\sum_{i=1}^{10} P_{i}
$$

onde, CC é o Coeficiente de Comprometimento e $\mathrm{P}_{\mathrm{i}}$ são as perguntas contidas no questionário com o intuito de compor o referido coeficiente.

Da mesma forma, propõe-se o coeficiente de Satisfação:

$$
\mathbf{C S}=\sum_{i=11}^{20} P_{i} \quad \mathbf{0} \leq \mathbf{C S} \leq \mathbf{1 0}
$$

onde, CS é o Coeficiente de Satisfação e $\mathrm{P}_{\mathrm{i}}$ são as perguntas contidas no questionário com o intuito de compor o referido coeficiente.

Assim, a soma das dez perguntas garante coeficientes que podem apontar respondentes com um coeficiente que varia entre um valor igual a zero (com baixíssimo comprometimento/satisfação) até respondentes que possuiriam um coeficiente igual a dez (com elevado comprometimento/satisfação com seu trabalho).

É importante ressaltar que no momento de respostas ao questionário, os indivíduos não tinham o conhecimento da ordenação de respostas e nem mesmo de que seria formulado algum tipo de coeficiente ordenador. Tal procedimento foi importante com o intuito de se garantir a minimização de vieses.

\subsection{PROCEDIMENTOS DE ANÁLISE}

Para realizar a análise dos dados coletados, adotaram-se três estratégias. Na primeira, utilizou-se uma análise descritiva através de Figuras que ilustram a relação entre variáveis importantes, tais como: idade, escolaridade, natureza do cargo e tempo de serviço com comprometimento e satisfação.

Em seguida foram apresentadas as principais estatísticas descritivas com as variáveis do modelo, relacionadas às características sociais predominantes. São apresentadas estatísticas de média, moda, valor máximo, valor mínimo e desvio padrão para cada uma das variáveis produzidas a partir do questionário proposto. Nunca é demais salientar que no caso das variáveis categóricas, a saber: local de residência, gênero, escolaridade e estado civil, são apresentadas as estatísticas das variáveis dummies que as representam.

Por fim, a terceira estratégia visava entender a provável relação entre comprometimento e satisfação. Para isso, foram feitas regressões lineares múltiplas.

Este modelo permite conhecer a relação entre as variáveis estudadas, além de sintetizar os inúmeros valores obtidos em uma única função. Como se sabe, a regressão linear múltipla é uma técnica multivariada cuja finalidade principal é obter uma relação matemática entre uma das variáveis estudadas (variável dependente ou resposta) e o res- 
tante das variáveis que descrevem o sistema (variáveis independentes ou explicativas), e reduzir um grande número de variáveis para poucas dimensões com o mínimo de perda de informação, permitindo a detecção dos principais padrões de similaridade, associação e correlação entre as variáveis. Ela ainda poderia ser usada na predição de resultados, por meio da regra estatística dos mínimos quadrados (SASsi et al, 2011).

É importante ressaltar que embora a análise de regressão lide com a dependência de uma variável em relação a outras variáveis, ela não implica necessariamente em causa. Uma relação estatística, por mais forte e sugestiva que seja jamais pode estabelecer uma relação causal. As ideias sobre causa devem vir de fora da estatística, enfim, de outra teoria.

No caso do modelo de regressão múltipla permite estimar o efeito da variação em uma variável $\left(X_{i i}\right)$ sobre $Y_{i}$ mantendo constantes ou outros regressores $\left(\mathrm{X}_{2 \mathrm{i}^{\prime}}, \mathrm{X}_{3 \mathrm{i}}\right.$ e assim por diante), conforme elucida Stock e Watson (2004).

No caso deste trabalho, estimar-se-á o seguinte modelo teórico:

$$
\mathrm{Y}_{\mathrm{i}}=\beta_{\mathrm{o}}+\beta_{1} \mathrm{X}_{1 \mathrm{i}}+\beta_{2} \mathrm{X}_{2 \mathrm{i}}+\ldots+\beta_{\mathrm{k}} \mathrm{X}_{\mathrm{ki}}+\mathrm{u}_{\mathrm{i}^{\prime}} \quad \mathrm{i}=1, \ldots, 68 .
$$

onde $Y_{i}$ é a i-ésima observação da variável dependente (CC ou $\mathrm{CS}) ; \mathrm{X}_{1 i^{\prime}} \mathrm{X}_{2 \mathrm{i}^{\prime}} \ldots, \mathrm{X}_{\mathrm{ki}}$ são as i-ésimas observações sobre cada um dos $\mathrm{k}$ regressores e $u_{i}$ é o termo de erro que captura o efeito de outras variáveis importantes não contidas no modelo.

\section{RESULTADOS}

São apresentadas inicialmente algumas relações entre as principais variáveis determinantes do Comprometimento e da Satisfação dos Servidores do IFPA - Campus de Santarém. Primeiramente, apresenta-se a relação existente entre os Coeficientes de Satisfação e de Comprometimento declarado pelos respondentes, na figura 1. Nesta figura são plotadas as combinações dos coeficientes dos 68 respondentes.

FIGURA 1 - Coeficiente de Satisfação e de Comprometimento dos Servidores do IFPA - Campus de Santarém.

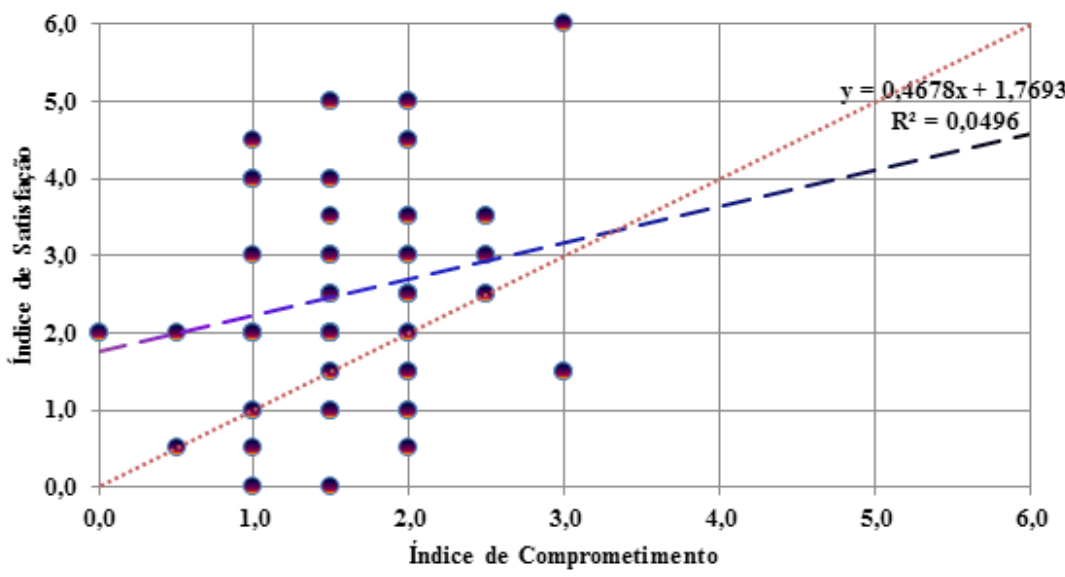

FONTE: Dados da Pesquisa 
Observando a figura 1, verifica-se que existem apenas 33 pontos e não 68 pontos como eram de se esperar em função do tamanho populacional. Acontece que em função da simplicidade dos Coeficientes formulados, muitos indivíduos tiveram coincidência nas combinações. O caso mais notável de todos foi à combinação de indivíduos que apresentaram um coeficiente de Comprometimento igual a 1,5 e um coeficiente de Satisfação equivalente a dois, tal combinação se repetiu entre sete respondentes.

Verifica-se que 12 respondentes revelaram ter um coeficiente de Comprometimento maior que o de Satisfação. Por outro lado, 47 respondentes revelaram o contrário e apenas nove revelaram igualdade entre os coeficientes. A linha pontilhada define o conjunto de combinações. Os pontos abaixo dessa linha são aqueles que revelam ter um coeficiente de Comprometimento maior que o de Satisfação e, vice-versa. Sob essa ótica, pode-se afirmar que, comparativamente, os servidores apresentaram maior satisfação que comprometimento organizacional.

Outra maneira mais detalhada de verificar a relação existente entre ambos os coeficientes é observando a figura 2. Esta figura apresenta conjuntamente os dois coeficientes e a diferença entre eles para toda a população estudada.

FIGURA 2 - Coeficientes de Comprometimento e de Satisfação e as suas respectivas diferenças para o conjunto populacional do IFPA - Campus Santarém

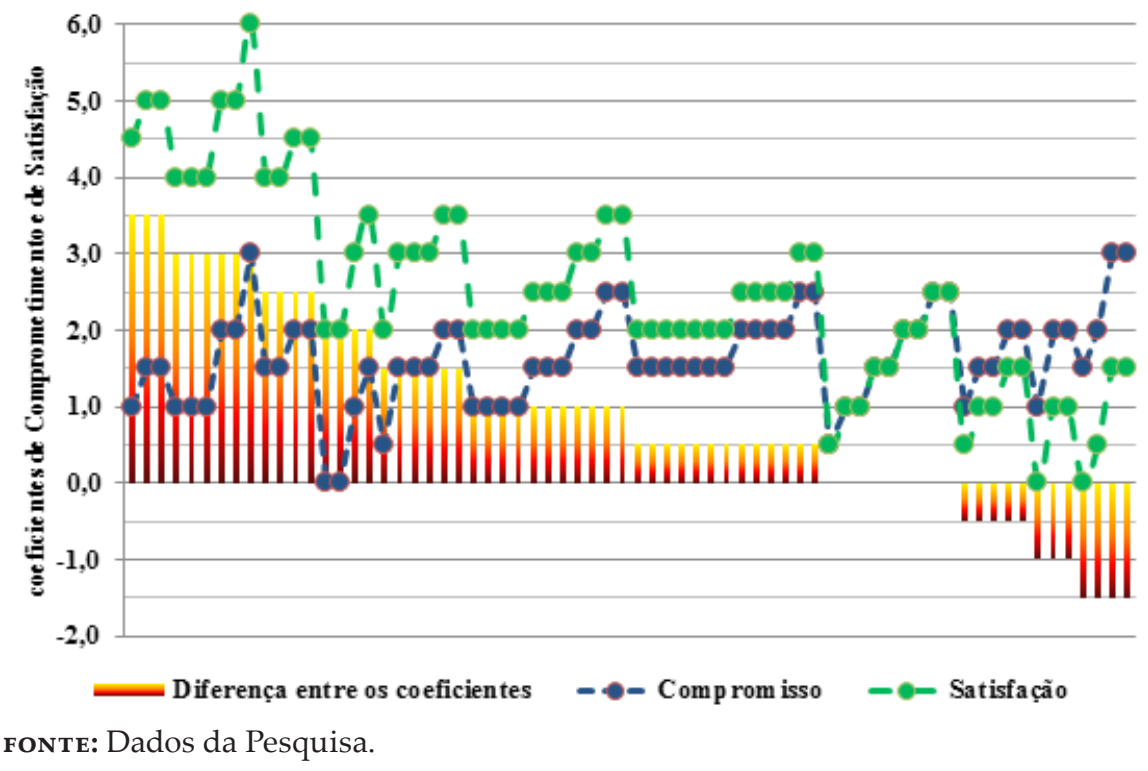

A figura 2 está ordenada pela maior diferença entre o coeficiente de Satisfação e o coeficiente de Comprometimento até a menor diferença entre eles. Verifica-se então que, por exemplo, três respondentes apresentam uma diferença entre esses dois coeficientes no valor de 3,5 (facilmente verificado no canto superior esquerdo) e que somente quatro respondentes apresentaram um valor de $-1,5$ (também facilmente verificado no canto inferior direito). 
Observando mais detalhadamente os valores dos dois coeficientes, percebe-se uma clara consonância entre ambos. Feito o cálculo de correlação entre os dois coeficientes, chega-se a um valor igual a 0,223.

Outra importante relação a ser verificada concerne à diferença desses coeficientes entre servidores homens e mulheres lotadas no IFPA - Campus de Santarém. A figura 3 apresenta as médias dos coeficientes para os dois grupos populacionais.

FIGURA 3 - Coeficientes de Compromisso e de Satisfação para os grupos populacionais femininos e masculinos.

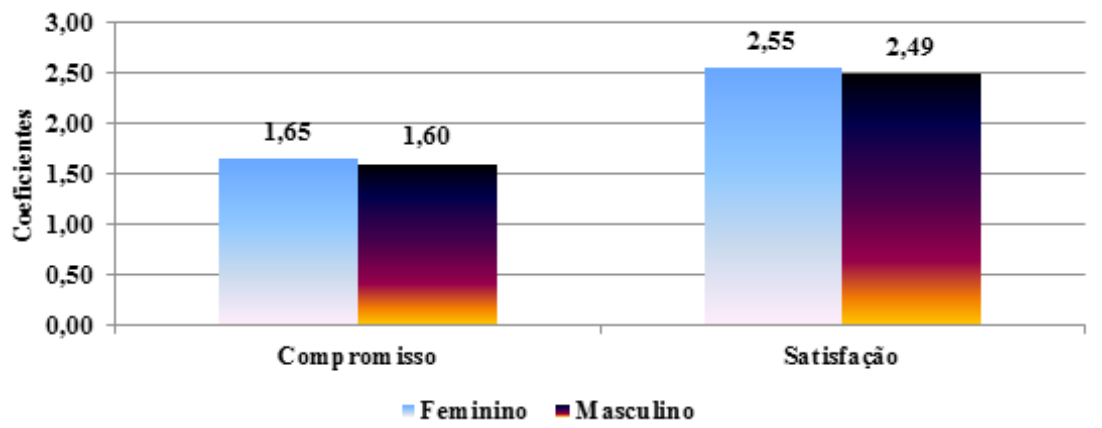

FONTE: Dados da Pesquisa.

Conforme se verifica as diferenças são mínimas entre os dois grupos. Observa-se que as diferenças somente podem ser verificadas quando observamos a segunda casa decimal dos coeficientes. Entretanto, de maneira geral, as mulheres apresentam valores superiores aos dos homens de maneira quase que inobservável. Alguns estudos sugerem que o gênero pode afetar a percepção de empregados em relação a comprometimento organizacional (MATHIEU; ZAJAC, 1990; SCANDURA; LANKAU, 1997;). Contudo, uma significativa parte de estudos não se mostram conclusivos nesse respeito.

Com relação à idade o que se verifica é que à medida que o tempo de vida dos servidores avança, os coeficientes médios se afastam. A figura 4 apresenta os coeficientes de Comprometimento e de Satisfação em relação às idades dos respondentes.

FIgURA 4 - Coeficientes de Comprometimento e de Satisfação em relação às idades dos respondentes

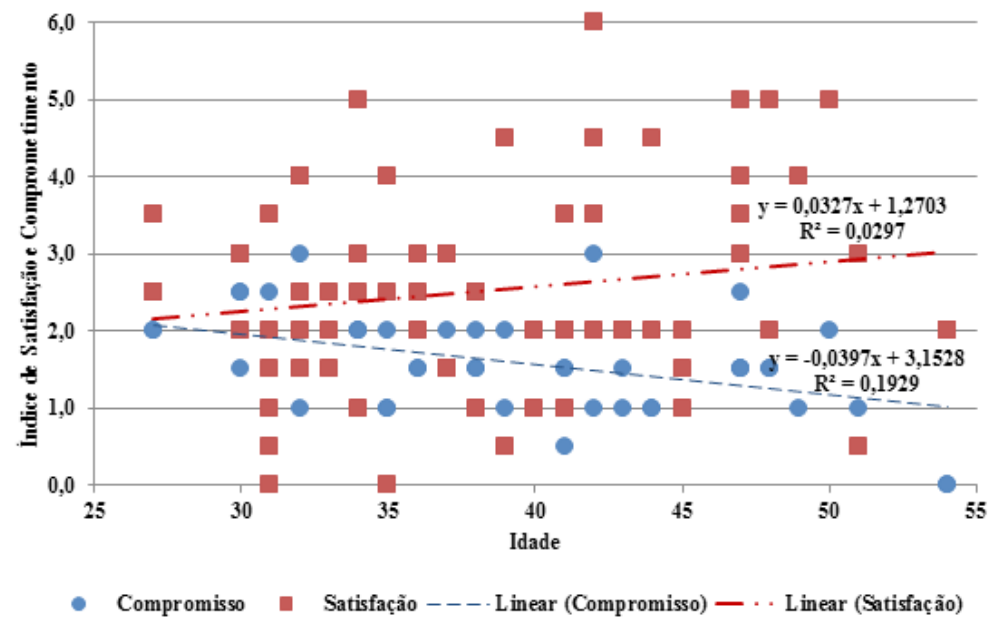

FONTE: Dados da Pesquisa. 
Observando a figura 4 se verifica que os coeficientes médios de Comprometimento e de Satisfação se afastam à medida que o servidor é mais velho.

Observe que a nuvem de pontos do coeficiente de Satisfação se torna bem mais alta à medida que se pergunta aos servidores mais velhos e o contrário acontece com o coeficiente de Compromisso. Este fato talvez se deva em razão de que os servidores com mais idade tenham tendência a mostrar mais satisfação em função da estabilidade, remuneração e possibilidade de carreira, entre outros, que o serviço público proporciona fatores que nem sempre são valorizados no serviço privado.

Esta tendência se verifica em Cortês e Silva (2006) que sugere algumas razões para a busca no emprego público, como menor discriminação no processo seletivo, no que tange a idade, como, por exemplo, não exigência de experiência prévia ou critérios estereotipados - como a aparência, redução de defasagem salarial.

Observando os dados, verifica-se que dois servidores tiveram coeficientes de Compromisso igual a zero e, neste caso ambos tinham a mesma idade, 54 anos. Observe ainda que na figura é apresentado um ajustamento linear de ambas as nuvens. As referidas linhas confirmam a impressão visual de afastamento dos coeficientes médios.

Com relação aos coeficientes relacionados aos níveis educacionais dos servidores, verificam-se algumas particularidades. A figura 5 apresenta os coeficientes de Comprometimento e de Satisfação em relação aos níveis de formação educacional dos servidores.

FIGURA 5 - Coeficientes de Comprometimento e de Satisfação em relação aos níveis de formação educacional dos servidores

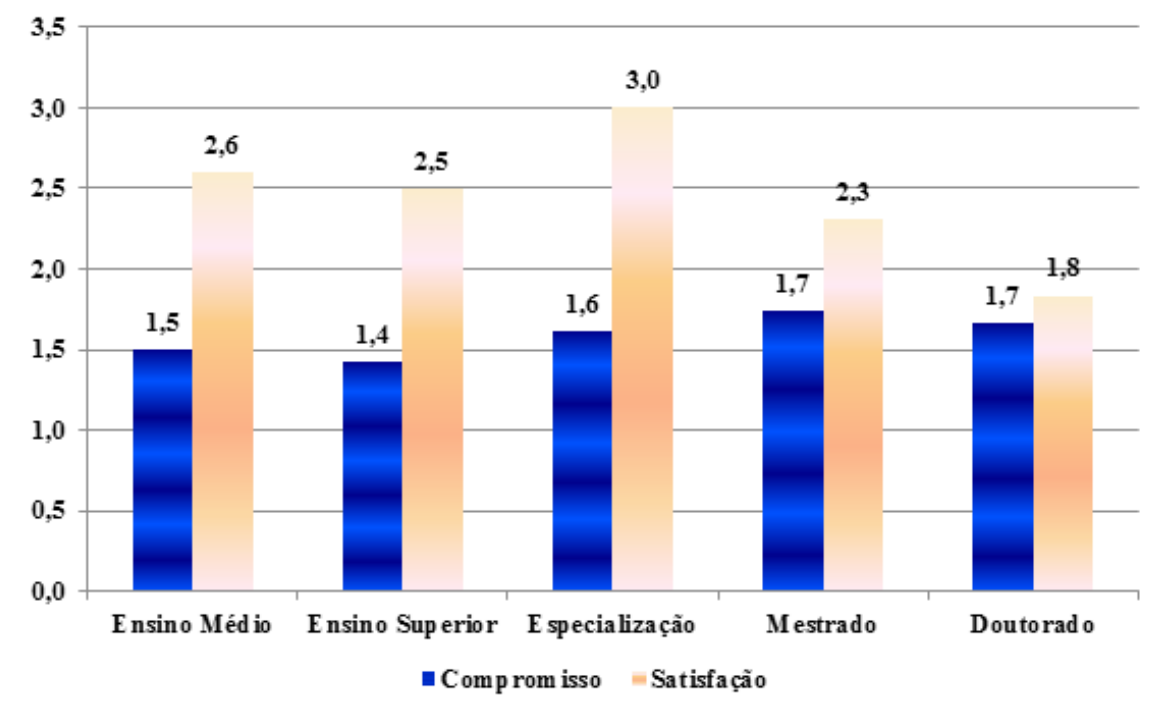

FONTE: Dados da Pesquisa.

A figura 5 apresenta fatos interessantes. Em primeiro lugar, independente do nível de escolaridade, os servidores se mostram mais satisfeitos que comprometidos. Segundo, os maiores coeficientes de Satisfação foram observados entre os níveis de ensino médio e especialização, enquanto que os menores coeficientes entre mestres e doutores. 
Verifica-se que a escolaridade se apresenta de forma diferente em relação a comprometimento e satisfação. Servidores com mais escolaridade se apresentaram mais insatisfeitos, por outro lado esse mesmo grupo apresentou o maior índice de comprometimento. Os índices de comprometimento seguem uma escala (quase que) hierárquica com a escolaridade.

Tal resultado corrobora com pesquisa de Brandão et al (2014) que mostra a relação entre escolaridade e o nível de satisfação com as atividades desempenhadas. O referido trabalho mostra que há uma menor satisfação quando se possui maior escolaridade. Neste mesmo trabalho, observa-se que quanto mais elevado o nível de escolaridade maior será o comprometimento.

A figura 6 apresenta os coeficientes de Comprometimento e de Satisfação para Técnicos Administrativos e Docentes do IFPA- Campus Santarém.

FIgURA 6 - Coeficientes de Comprometimento e de Satisfação para Técnicos Administrativos e Docentes do IFPA-Campus Santarém

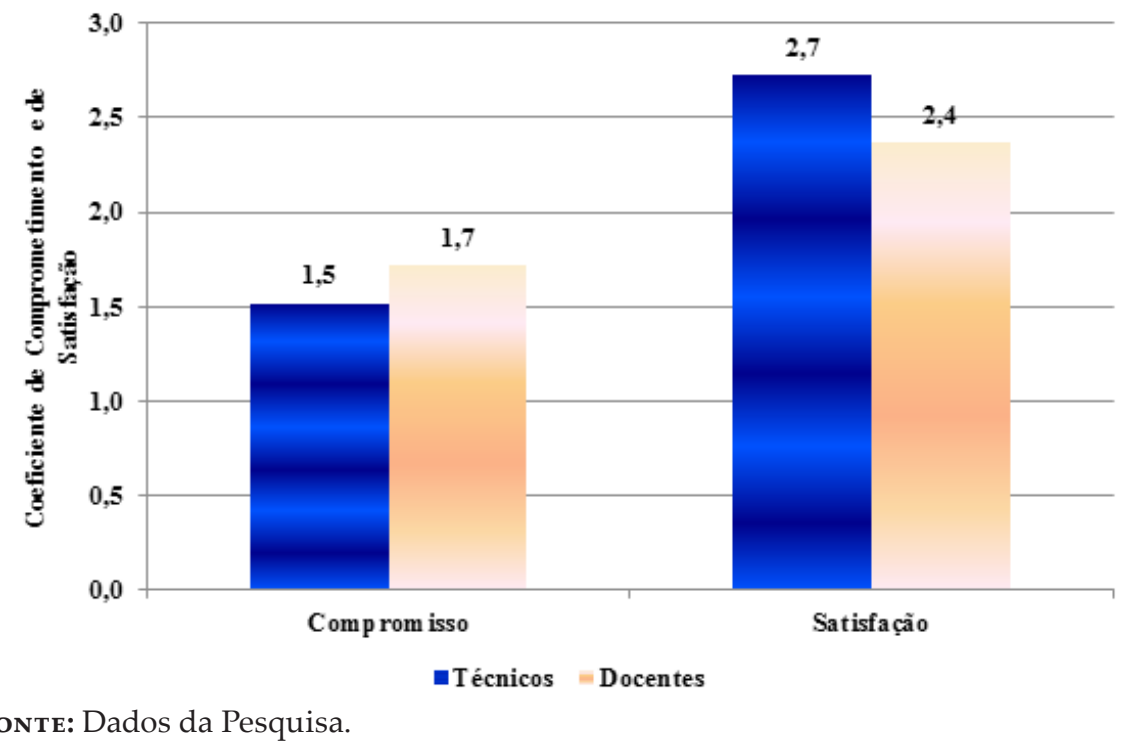

Na figura 6, verifica-se que em função da natureza do cargo ocupado, os docentes mostraram mais comprometimento enquanto que os técnicos mais satisfação, contudo a diferença entre esses índices não foi significativa em nenhuma das variáveis. É provável que fatores que contribuem para o desenvolvimento do comprometimento organizacional e satisfação no trabalho, como o vínculo emocional associado à ideia de identificação, de lealdade, de orgulho e desejo de pertencer, bem como sentimento de dever, a partir da identificação e internalização dos valores e objetivos da instituição (BASTOS, BRANDÃO E PINHO, 1997), sejam percebidos da mesma maneira tanto pelos docentes como pelos técnicos.

Por fim, A figura 7 apresenta os coeficientes médios de Satisfação e Comprometimento por tempo de serviço dos servidores do IFPA Campus Santarém. Observe que no período da pesquisa de campo o tempo máximo de serviço dos servidores era restrito a um intervalo entre um e cinco anos de serviço. 
FIGURA 7 - Coeficientes médios de Satisfação e Comprometimento por tempo de serviço dos servidores do IFPA - Campus Santarém.

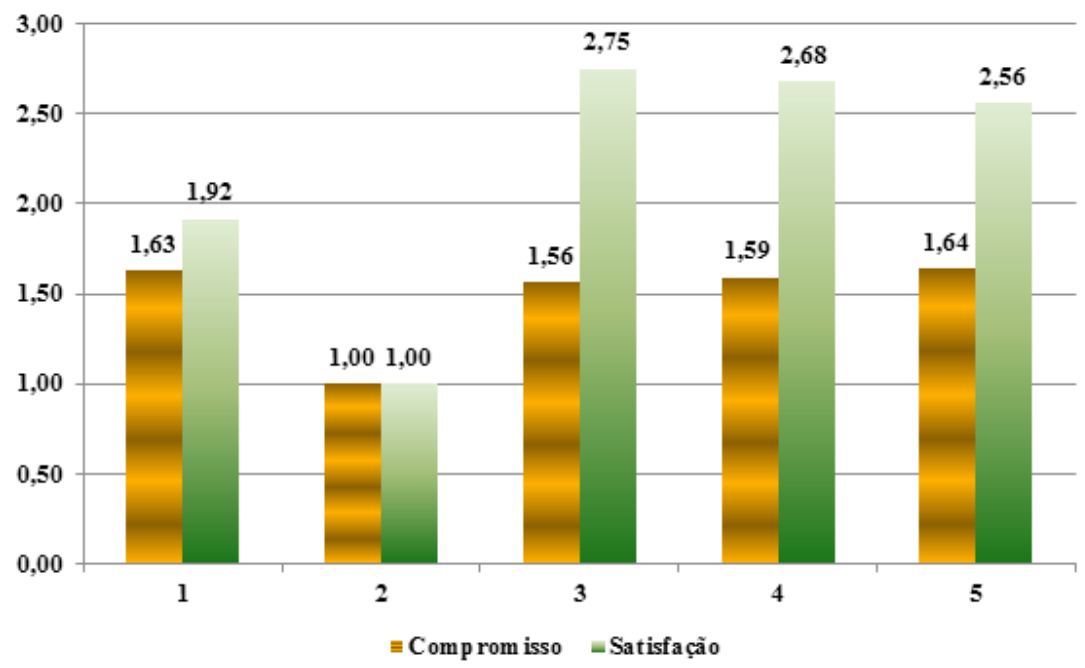

FONTE: Dados da Pesquisa.

A referida figura mostra que há um salto significativo no Coeficiente médio de Satisfação a partir dos servidores que completaram três anos de serviço no IFPA, enquanto que o Coeficiente de Comprometimento médio se mostra praticamente inerte em todo o período. Talvez esses números evidenciem um aumento de satisfação enquanto o servidor passa mais tempo engajado no serviço. Por outro lado, com relação ao comprometimento, não parece haver grandes variações.

\subsection{ESTATÍSTICAS DESCRITIVAS}

A tabela 1 apresenta as estatísticas descritivas do modelo. $\mathrm{O}$ objetivo desta tabela é o de se ter uma visão mais geral das principais características da população estudada.

TABELA 1 - Estatísticas descritivas das principais variáveis do modelo

\begin{tabular}{c|c|c|c|c|c}
\hline ESTATÍSTICAS & MÉDIA & MODA & MÁxIMO & MÍNIMO & DESVIO PADRÃO \\
$\begin{array}{c}\text { Coeficiente de } \\
\text { Compromisso }\end{array}$ & 1,62 & 1,5 & 3 & 0 & 0,63 \\
\hline $\begin{array}{c}\text { Coeficiente de } \\
\text { Satisfação }\end{array}$ & 2,53 & 2 & 6 & 0 & 1,31 \\
\hline Local de Residência & 0,91 & 1 & 1 & 0 & 0,29 \\
\hline Gênero (Homem) & 0,54 & 1 & 1 & 0 & 0,50 \\
\hline Idade & 38,5 & 31 & 54 & 27 & 6,92 \\
\hline Escolaridade & 2,18 & 3 & 4 & 0 & 1,04 \\
\hline Função & 0,54 & 1 & 1 & 0 & 0,50 \\
\hline Tempo de serviço & 4,21 & 5 & 5 & 1 & 1,25 \\
\hline (Anos) & 0,62 & 1 & 1 & 0 & 0,49
\end{tabular}

FONTE: Dados da Pesquisa. 
Conforme se verifica na Tabela 1 são apresentadas cinco estatísticas que representam as características predominantes da população de docentes e técnicos administrativos do IFPA, Campus Santarém, objeto deste estudo. Nunca é demais informar que no caso das variáveis categóricas, a saber: local de residência, gênero, escolaridade e estado civil, são apresentadas às estatísticas das variáveis dummies que as representam.

O coeficiente de Compromisso médio dos entrevistados foi de 1,62. Observe que, analogamente, o coeficiente de Satisfação foi de 2,53. Assim, a partir do indicador proposto e da coleta de dados, pode-se afirmar que os servidores revelam ter muito mais Satisfação com seu trabalho do que Comprometimento. Resultado semelhante também foi verificado em trabalho como o de Souza, Reche e Sachuk (2013).

A explicação para o menor comprometimento em relação à satisfação pode ser explicada por Borges-Andrade \& Pilati (1999), citados no trabalho de Kunkel e Vieira (2012). Os autores explicitaram que as disfunções típicas do setor público, decorrentes de um aparato burocrático excessivo, geram baixos padrões de comprometimento organizacional e envolvimento com o trabalho.

Seguindo na mesma linha, verifica-se que o valor de coeficiente de Compromisso mais vezes verificado foi o de 1,5 , enquanto que o mais repetido, com relação à Satisfação, foi o valor de 2 referentes às estatísticas moda. Verifica-se ainda que o maior coeficiente de Satisfação entre os entrevistados foi duas vezes maior que o maior coeficiente de Comprometimento. Enquanto que, com relação à dispersão dos valores populacionais, o coeficiente de Satisfação apresentou uma dispersão duas vezes maior que o coeficiente de Compromisso, conforme se verifica os valores de Desvio Padrão dessas duas variáveis.

Com relação a gênero, verifica-se que $54 \%$ dos servidores lotados no IFPA - Campus Santarém, é composto por homens.

A idade média dos respondentes fica em torno dos 38 anos de idade o que representa uma população ainda muito jovem com a possibilidade de contribuir com o sucesso daquela Instituição por muito tempo. Verificou-se ainda que o servidor mais jovem tinha apenas 27 anos, enquanto que o mais velho 54 anos. Verifica-se também, que a idade mais comum entre os respondentes é 31 anos de idade.

Em relação à escolaridade, verifica-se que o Campus, ao período da pesquisa, apresentou uma maior frequência de mestrado. Se por um lado, isso pode significar maior possibilidade de melhor contribuição, por outro, pode significar também, maior nível de exigência em relação à instituição, bem como a satisfação, pois quanto maior escolaridade, maior será seu nível de exigência para satisfação e quanto menor escolaridade menor será seu nível de exigência para satisfação. Isso se verifica no trabalho de Brandão et al (2014), cujo resultado mostrou menor satisfação quando se possui maior escolaridade.

Com relação à natureza do cargo, os resultados mostram que $54 \%$ dos servidores são docentes e $46 \%$ técnico administrativo. 
No que tange ao tempo de serviço na instituição, ressalta-se que a maior parte dos pesquisados (42\%) trabalha há cinco anos no Campus, ou seja, a maior parte dos servidores está no Campus desde a sua inauguração. O Campus Santarém teve suas atividades iniciadas em novembro de 2010.

Em linhas gerais, percebe-se que o Campus Santarém como um Campus recentemente criado, com apenas cinco anos de atividades no munícipio, conta com servidores jovens e possuem um grau de escolaridade razoavelmente elevado. A Instituição tem em seu quadro dois cargos, sendo um da categoria técnico administrativo e outro da categoria docente e que no momento da pesquisa, o número de docentes era maior que o número de técnicos.

\subsection{RESULTADOS DAS REGRESSÕES}

Os resultados obtidos das regressões para os modelos que relacionam as variáveis observáveis dos servidores com a Satisfação e o Comprometimento no trabalho dos servidores do IFPA - Campus de Santarém - serão apresentados.

O objetivo das regressões lineares múltiplas é o de verificar quais características, facilmente observáveis e mensuráveis a partir de respostas ao questionário, estão mais relacionadas com os Coeficientes de Comprometimento e de Satisfação calculadas a partir do modelo proposto anteriormente nesse trabalho.

A tabela 2 apresenta os resultados desses dois modelos.

TABELA 2 - Variáveis relacionadas à Satisfação e Comprometimento dos Servidores lotados no IFPA - Campus Santarém

\begin{tabular}{|c|c|c|c|c|}
\hline \multirow{2}{*}{ VARIÁVEL } & \multicolumn{2}{|c|}{ COMPROMETIMENTO } & \multicolumn{2}{|c|}{ SATISFAÇÃO } \\
\hline & COEFICIENTE & SIGNIFICÂNCIA & COEFICIENTE & SIGNIFICÂNCIA \\
\hline Constante & 3,804 & 0,000 & $-3,024$ & 0,129 \\
\hline Comprometimento & - & - & 0,809 & 0,006 \\
\hline Satisfação & 0,147 & 0,006 & - & - \\
\hline Idade & $-0,046$ & 0,000 & 0,051 & 0,057 \\
\hline Escolaridade & $-0,870$ & 0,061 & 1,522 & 0,165 \\
\hline Natureza do Cargo & 0,261 & 0,126 & $-0,719$ & 0,072 \\
\hline Tempo de Serviço & $-0,225$ & 0,137 & 0,522 & 0,141 \\
\hline Estado Civil & 0,218 & 0,119 & 0,323 & 0,329 \\
\hline Escol*TempServ & 0,187 & 0,063 & $-0,312$ & 0,190 \\
\hline $\mathrm{N}$ & \multicolumn{2}{|c|}{68} & \multicolumn{2}{|c|}{68} \\
\hline $\mathrm{R}^{2}$ & \multicolumn{2}{|c|}{0,214} & \multicolumn{2}{|c|}{0,372} \\
\hline Teste F & \multicolumn{2}{|c|}{$\begin{array}{c}2,341 \\
(0,035)\end{array}$} & \multicolumn{2}{|c|}{$\begin{array}{c}5,081 \\
(0,000)\end{array}$} \\
\hline Durbin-Watson & \multicolumn{2}{|c|}{2,093} & \multicolumn{2}{|c|}{1,800} \\
\hline
\end{tabular}

FONTE: Dados da Pesquisa. 
O primeiro modelo relaciona cinco perfis sociais, a saber: idade, escolaridade, natureza do cargo, tempo de serviço e estado civil. Relaciona-se ainda, com uma variável interativa entre Escolaridade e Tempo de Serviço. O segundo modelo, utiliza-se das seis variáveis mencionadas e ainda, o Coeficiente de Comprometimento.

A partir do modelo apresentado que explica as variáveis que mais se relacionam com o Comprometimento dos servidores, verifica-se que quatro das sete variáveis têm significância estatística a 90\%.

Inicialmente, chama atenção a correlação existente entre Comprometimento e Satisfação. Os resultados mostram que o impacto do aumento da Satisfação no Comprometimento é na ordem de $15 \%$, dentro dos limites impostos pelo modelo. Esse resultado faz pensar que há uma interação entre essas duas características e que elas interagem na mesma direção. Assim, quem está mais satisfeito termina assumindo maior comprometimento. Este resultado encontra similaridade de resultados com o trabalho de Kunkel e Vieira (2012), que utilizando o coeficiente de correlação de Spearman concluíram que quanto maior o nível de envolvimento do indivíduo, maior será o seu comprometimento e a sua satisfação com salário, promoções, tarefas, relacionamento com colegas e chefia.

Em seguida, verifica-se que a idade guarda uma relação negativa e estatisticamente significante com o Coeficiente de Comprometimento dos servidores do IFPA - Campus Santarém. O estimador de idade mostra que a cada ano, em média, os servidores diminuem o seu comprometimento em uma taxa de 4,6\%. Este resultado talvez esteja captando um importante efeito: a estrutura pouco motivacional da carreira pública no Brasil. O instituto da estabilidade no serviço público, a ausência de incentivos à produtividade, dentre outras características endêmicas da carreira pública no Brasil, cria um ambiente desmotivador e impróprio para um sentimento de comprometimento. Com o passar dos anos o jovem servidor começa a entender melhor esses mecanismos, internalizá-los e perde o interesse. O seu comprometimento passa a ser mais cartorial e menos visceral. Tal resultado encontra similaridade com os trabalhos de Botelho \& Paiva (2011), em pesquisa realizada com 330 servidores do Tribunal de Justiça do Estado de Minas Gerais. Os autores concluíram que os servidores se encontravam descomprometidos em função de falta de infraestrutura, sobrecarga de trabalho, escassez de perspectivas no plano de carreira e, principalmente, devido à ausência de valorização do trabalho.

Outro resultado interessante é o da escolaridade. O impacto negativo da escolaridade surpreende em certa medida. Era de se esperar um comprometimento maior daqueles que, por hipótese, tem um maior protagonismo em função de sua maior escolaridade. Entretanto, o resultado que se vê é o de que aqueles que têm menor escolaridade tem menor comprometimento, controlado pelas demais características do modelo. É importante ressaltar que esse resultado se inverte quando a escolaridade está interagindo com o tempo de serviço, conforme será 
detalhado em seguida. Resultado parecido foi percebido no trabalho de Pinto (2011) utilizando a técnica intitulada CHAID - Chi-Square Automatic Interaction Detector. O trabalho avaliou o nível de comprometimento e desempenho do técnico administrativo por carreiras (A B C D E), que no serviço público federal, na área de educação, representa A, B , C - nível médio de escolaridade e, D e E - nível de escolaridade superior. Verificou-se que o desempenho de funcionários das carreiras de nível médio apresentava média inferior aos indivíduos de nível superior.

Por fim, a variável "Escol*TempServ" que não é nada mais do que a interação entre a Escolaridade e o tempo de serviço do servidor, apresenta um efeito positivo e estatisticamente significante. Pode-se entender que à medida que se elevam a escolaridade e o tempo de serviço, os servidores encontram maiores razões ou mesmo maiores responsabilidades e revelam maior comprometimento.

Assim, dentre as variáveis estudadas, pode-se afirmar que dentre os servidores lotados no IFPA - Campus de Santarém, aqueles que revelam maior comprometimento com aquela Instituição são aqueles que revelam maior satisfação com o trabalho, os mais jovens, àqueles que têm menor escolaridade e aqueles que, em tendo maior escolaridade também tem maior tempo no serviço público.

Com relação ao Coeficiente de Satisfação, verifica-se que dentre as oito variáveis testadas apenas três são estatisticamente significantes, a saber: Coeficiente de Comprometimento, idade e docentes.

Em relação ao coeficiente de Comprometimento, verifica-se que o impacto se mostrou positivo e elevado. Uma elevação de um ponto no coeficiente de Comprometimento traz a elevação, em média de $81 \%$ no coeficiente de Satisfação do servidor. Este resultado parece trazer evidências de que a Satisfação está, em boa medida, no comprometimento dos servidores. É possível imaginar que pessoas mais comprometidas encontram maior satisfação quando conseguem ver maior significado em seu esforço laboral. Conseguem contemplar os resultados práticos de seu esforço. Elas se sentem parte do processo e tomam para si os resultados verificados. Este resultado encontra similaridade de resultados com os trabalhos de Kunkel e Vieira (2012) que utilizando a Matriz de correlação de Spearman, afirmam que os servidores sentem-se ligados ao trabalho e acreditam que o ambiente laboral propicia crescimento e satisfação pessoal, o que, segundo Siqueira \& Gomide Júnior (2004), contribui para o desenvolvimento e a manutenção de motivação, dedicação e comprometimento para com a organização.

Outra característica importante é a idade. Ao contrário do que se viu no modelo anterior, à medida que os anos se passam para os servidores eles encontram maior satisfação no trabalho. Este resultado talvez tenha a ver a com as características da carreira pública no Brasil que traz estabilidade e diminui as incertezas futuras dos servidores. É sabido que pessoas mais velhas têm maior preferência pela estabilidade. Ainda mais porque à medida que os anos se passam elas se sentem 
mais protegidas das incertezas, assim, tendem a se estabelecer em certas zonas de conforto, que por outro lado, pode trazer uma diminuição de sua competitividade no mercado de trabalho - o que realimenta o processo de satisfação. Este resultado encontra similaridade com o trabalho de Pedro e Peixoto (2006), que através da análise de variância, identificaram que um grupo de professores mais antigos apresentou nível de satisfação maior que professores mais novos.

Por fim, com relação aos fatores correlacionados com o coeficiente de Satisfação, tem-se a diferenciação entre docentes e técnicos administrativos. O modelo estima que os docentes tenham $28 \%$ menos de satisfação quando comparado com os técnicos administrativos. Este resultado talvez esteja relacionado com as condições de trabalho e oportunidades consideradas pelos docentes como insuficientes. Nos novos Campi da recente expansão do ensino superior e técnico no Brasil não é incomum se ouvir sobre as condições ainda inapropriadas para a realização de pesquisas e o bom desenvolvimento de atividades correlatas. Em se considerando que os docentes têm maiores dependências dessas condições para a realização de seu trabalho, não é difícil imaginar que eles tenham maiores razões para tal insatisfação. Este resultado encontra similaridade de resultados com os trabalhos de Gursel, Sunbul e Sari (2002) e, também, Scoot, Cox e Dinham (1999) apud Pedro e Peixoto (2006) que identificaram que a satisfação profissional progride de forma inversa aos anos de carreira, sendo que os professores mais velhos é quem evidencia menor satisfação no trabalho. No final da década de 6o, Sergiovanni (1967, cit. por Trigo-Santos, 1996) aplicou a teoria e metodologia de Herzberg a professores americanos e concluiu que a tendência expressa nos resultados corroboravam o que Herzberg havia postulado: a satisfação dos professores derivada de fatores relacionados com a docência em si, enquanto que o descontentamento decorria sobretudo das condições sócio-políticas de trabalho. Segundo Pedro e Peixoto (2011), estudos posteriormente desenvolvidos com professores de outras nacionalidades têm espelhado a mesma tendência nesses resultados (Bellot \& Tutor, 1990; Cordeiro-Alves, 1991; Kaufman, 1984; Moreno, 1995; Trabulho, 1999).

Assim, verifica-se que o coeficiente de Satisfação é explicado a partir dessas três características: coeficiente de Comprometimento, idade e docente. Pode-se afirmar que servidores com maior comprometimento institucional, mais velho e não docentes, encontram maiores razões para maior satisfação no IFPA Campus de Santarém. 


\section{CONCLUSÃO}

O presente estudo teve por objetivo estudar o comportamento dos servidores do Instituto Federal do Pará - campus Santarém - em relação a comprometimento organizacional e satisfação no trabalho.

O principal resultado encontrado foi a forte evidência da existência de correlação entre comprometimento e satisfação, na mesma direção. Assim, pode-se afirmar que quem está mais satisfeito está também mais comprometido no IFPA - Campus Santarém. Tal resultado não espanta e traz consigo uma forte percepção intuitiva: indivíduos insatisfeitos tendem a encontrar grandes dificuldades de comprometimentos em seu labor.

Com relação ao comprometimento organizacional, verificou-se que fatores como idade, escolaridade e a interação entre Escolaridade e Tempo de Serviço estão mais fortemente relacionados a esta percepção. Com relação à idade se verificou que os mais jovens revelam menor comprometimento. Foi verificada uma relação negativa entre comprometimento e escolaridade que parece ter alguma relação com eventuais frustrações dos quadros mais bem qualificados que se decepcionam ao não encontrar, eventualmente, o ambiente laboral almejado durante $\mathrm{o}$ longo processo de formação.

Com relação à satisfação do servidor, verifica-se que servidores mais velhos são os mais satisfeitos. Não causa nenhuma perplexidade esse resultado. Uma vez que o servidor vai ficando mais velho ele tende a perder competitividade no mercado de trabalho e valorizando cada vez mais a estabilidade. Da mesma maneira, os servidores técnicos administrativos apresentam maior satisfação que os docentes. E, ainda com relação à satisfação do servidor, verifica-se que o que melhor explica a sua percepção é o seu comprometimento organizacional. Esse é o encerramento de um elo primoroso: Servidores mais comprometidos são aqueles mais satisfeitos, enquanto que servidores mais satisfeitos são aqueles mais comprometidos. Parece haver uma perfeita reciprocidade entre tais percepções.

Como sugestões para futuros trabalhos, recomenda-se incorporar outras variáveis importantes para melhor compreensão do fenômeno, propor uma forma funcional matemática para compreender de forma sistemática tais relações e replicar este experimento em corporações mais complexas. Pode-se ainda, fazer comparações entre corporações para compreender como essas características se modificam em função da complexidade do ambiente do trabalho e da competitividade no mercado laboral.

Por fim, tais conclusões podem ajudar a Instituição a promover ações que visem estratégias de gestão que aperfeiçoem a eficiência da administração pública via mecanismos de satisfação e comprometimento dos servidores. Em relação ao Campus Santarém, os resultados poderão nortear políticas de gestão estratégica de pessoas que venham promover maior comprometimento organizacional e satisfação no trabalho entre os servidores. 


\section{REFERÊNCIAS}

AL-AJMI, R. The Effect of Gender on Job Satisfaction and Organizational Commitment in Kuwait. International Journal of Management Vol. 23. n. 4. December 2006.

AMÂNCIO, L. A; et al. Avaliação da satisfação no trabalho na percepção de enfermeiros gestores de um hospital maternidade. Enciclopédia Biosfera - Centro Científico Conhecer, Goiânia, v.10, n.19, p.119-130, 2014.

BASTOS, A.V. B. O conceito de comprometimento: sua natureza e papel nas explicações do comportamento humano no trabalho. Organizações e Sociedade, 1(2), p77-106. 1993.

BASTOS, A.V.B.; BRANDÃO, M.G.A.; PINHO, A.P.M. Comprometimento organizacional: uma análise do conceito expresso por servidores universitários no cotidiano de trabalho. Revista de Administração Contemporânea - RAC, v. 1, n. 2, mai/ago. 1997.

BOTELHO, R. D.; PAIVA, K. C. M. Comprometimento organizacional: um estudo no Tribunal de Justiça do Estado de Minas Gerais. Revista de Administração Pública, v. 45, n. 5, p. 1.249-1.283, Rio de Janeiro.

BORGES-ANDRADE, J.E.; AFANASIEF, R.S.; SILVA, M.S. Mensuração e comprometimento organizacional em instituições públicas. In: XIX Reunião Anual De Psicologia. Programa e resumos..., Ribeirão Preto, SP: Sociedade de Psicologia de Ribeirão Preto, 1989.

BORGES-ANDRADE, Jairo Eduardo \& PILATI, Ronaldo. Comprometimento atitudinal e comportamental: relações com suporte e imagem nas organizações. In: XXIII Encontro da Associação Nacional de Pós-Graduação e Pesquisa em Administração ENANPAD. Anais... Foz do Iguaçu: Anpad, 1999.

BRANDÃO, M.G.A. Comprometimento organizacional na administração pública: um estudo de caso em uma instituição universitária. Dissertação de mestrado. Salvador, BA, Escola de Administração, UFBA, 1991.

BRANDÃO, I. D. F. et al. Satisfação no serviço público: um estudo na superintendência regional do trabalho e emprego do Ceará. Revista Eletrônica de Administração, Porto Alegre, v. 1, n. 77, p. 90-113, jan./abr, 2014. 
BRITO, J. M.; BRITO, V. G. P. Gestão Estratégica de

Recursos Humanos. Lavras: UFLA \FAEPE, 2000.

CODA, Roberto. Psicodinâmica na vida

organizacional. $2^{\underline{a}}$ edição, São Paulo, Atlas, 1997.

CORTES, Leonardo Lopes, SILVA Jose Roberto Gomes da. Construção do Contrato Psicológico de indivíduos que ingressam em organizações no setor público no atual contexto brasileiro. 2006, Salvador. Artigo disponível em www.anpad.org.brlenanpad $\backslash 2006$. Acesso em: 27.05.2016.

SILVA SOUZA, I. D.; MARTIM RECHE, P.; SACHUK, M. I. O Comprometimento Organizacional e a Satisfação no Trabalho em Funcionários de um Hospital da Cidade de Umuarama - PR. Qualitas Revista Eletrônica, [S.1.], v. 14, n. 1, jan. 2013. Disponível em: $<$ http://revista.uepb.edu.br/index. php/qualitas/article/view/1712/927>. Acesso em: 14 Jun. 2016.

DESSLER, G. Conquistando comprometimento: como construir uma força de trabalho competitivo. São Paulo: Makron Books, 1996.

DIAS, J.M.G.; MORAES, L.F.R. Preditores do comportamento organizacional na EMATER-MG.

Temas em Psicologia, Ribeirão Preto-SP, v. 2, n. 1, 1993.

ETZIONI, A. Organizações modernas. $7^{\text {a }}$ ed. São Paulo: Pioneira, 1984. 190 p.

GOMES, A. A. P.; QUELHAS, O. L. G. Motivação de recursos humanos no serviço público: um estudo de caso sob dois ângulos teóricos. REAd - Revista Eletrônica de Administração. V.9, n.5, p.1-18. 2003.

HERZBERG, F. O conceito de higiene como motivação e os problemas do potencial humano de trabalho, In Hampton, David R. (Comp.). Conceitos de comportamento na administração. São Paulo: EPU, 1973.

KUNKEL, Franciele Inês Reis. VIEIRA, Kelmara Mendes. BemEstar no Trabalho: Um Estudo Junto aos Servidores Públicos da Prefeitura Municipal de Cerro Largo, Rio Grande Do Sul, 2012.

LEITE, C. F. F. O comprometimento organizacional na gestão pública: um estudo de caso em uma universidade estadual. 214f. (Dissertação de Mestrado). Escola de Administração. Universidade Federal da Bahia - EFBA, Vitória da Conquista, 2004. 
MASLOW, A.H. Motivation and personality.

2 ed. New York, Harper \& Row, 1970.

MATHIEU, J.E., ZAJAC, D.M. A review and meta-analysis of the antecedents, correlates and consequences of organizational commitment. Psycological Bulletin, v. 108, n. 2, p. 171-194, 1990.

PAULI, J.; BIULCHI, A. F. Avaliação de desempenho no serviço público: a experiência do instituto nacional do seguro social na implantação da gratificação de avaliação de desempenho do seguro social. GDASS - Rev. de Administração IMED. V. 2, n.2, p.129-138. 2012.

PEDRO, Neuza; PEIXOTO, Francisco. Satisfação profissional e auto-estima em professores dos $2 .{ }^{\circ}$ e $3 .{ }^{\circ}$ ciclos do Ensino Básico. Análise Psicológica, p. 247-262, 2006.

PINTO, Marcelo Pádua Carvalho: Comprometimento organizacional: um estudo de suas relações com desempenho na carreira, 2011. Dissertação apresentada ao Curso de Mestrado da Faculdade de Ciências Empresariais da Universidade FUMEC.

PINTO, Michele Nagakami Braule. A importância da qualidade de vida no trabalho para o bem estar do colaborador. Artigo. Publicado em Psicologado.com, ed fev, 2015.

SCANDURA, Terri A., LANKAU, Melenie J. Relationships gender, family responsability and flexible work hours to organizational commitment and job satisfaction. Journal of Organizational Behavior. V. 18. 1997

TAMAYO, A.; PASCHOAL, T. A relação da motivação para o trabalho com as metas do trabalhador. RAC, v. 7, n. 4, p. 33-54, out./dez. de 2003.

TRIGO-SANTOS, F. (1996). Atitudes e crenças dos professores do ensino secundário: satisfação, descontentamento e desgaste profissional. Lisboa: Instituto de Inovação Educacional.

VILLORIA, M.; IGLESIAS, A. Leadership in public management: some theoretical and methodological considerations. Innovar, v.21, n.42, p.174-189, 2011. 